\title{
The role of structural biology in pandemic's puzzles: amino acids and short peptides as key players
}

\author{
Joanna Bojarska ${ }^{1 *}$, Vasso Apostolopoulos ${ }^{2}$, John Matsoukas ${ }^{3,4}$, Jack Feehan ${ }^{2}$, Harry Ridgway ${ }^{5,6}$, \\ Piotr Zielenkiewicz ${ }^{7,8}$, Wojciech M. Wolf ${ }^{1}$ \\ ${ }^{1}$ Department of Chemistry, Technical University of Lodz, Zeromskiego 116, 90-924 Lodz, Poland; \\ ${ }^{2}$ Institute for Health and Sport, Victoria University, Melbourne, VIC 3030, Australia; \\ ${ }^{3}$ NewDrug, Patras Science Park, 26500 Patras, Greece; \\ ${ }^{4}$ Department of Physiology and Pharmacology, Cumming School of Medicine, University of Calgary, Canada; \\ ${ }^{5}$ Institute for Sustainable Industries and Liveable Cities, Victoria University, Melbourne, Australia; \\ ${ }^{6}$ AquaMem Consultants, Rodeo, New Mexico, USA; \\ ${ }^{7}$ Institute of Biochemistry and Biophysics, Polish Academy of Sciences, Pawinskiego 5a, 02-106 Warsaw, Poland; \\ ${ }^{8}$ Department of Systems Biology, Institute of Experimental Plant Biology and Biotechnology, University of Warsaw, Miecznikowa 1, \\ 02-096 Warsaw, Poland;
}

joanna.bojarska@p.lodz.pl

Since one and a half years the world has been fighting a COVID-19 pandemic, which caused unprecedented crisis all over the world. And, the full evolutionary potential of SARS-CoV-2 has yet to be revealed. A super-virus with features of the highly transmissible SARS$\mathrm{CoV}-2$ and the deadly SARS and MERS viruses could lead to more catastrophic loss of life. What is more, a new serious onslaught due to (corona)viruses and other pathogens are inevitable. Structural biology has been at the centre of the efforts of development of effective therapeutics. It helps to „see” 3D protein structures of invisible viruses and their interactions with host proteins, and potential ligands, knowing molecular mechanisms driving the viral evolution and shape biomedicinal research field [1,2]. Only through structural biology can we gain a deeper insight into the new variants of viruses and effect of mutations on the proteins. The ongoing outbreak has pushed numerous structural studies, using crystallography, cryoelectron microscopy, structural virology and vaccinology, structural bioinformatics, dynamics, and multi-omics, leading to either revolutionary progress of structural biology and understanding of pandemic evolution leading to therapeutic findings. Here, we mention about some examples. The basic biomolecules, amino acids, and short peptides, as constituents of proteins associated with RNA, are crucial elements in the pandemic's puzzles. Amino acid variations in the spike of SARS-CoV-2 affects the shape, binding, and function of the protein. The virus can escape neutralizing antibodies through only a single amino acid replacement. Thanks to structural studies, we found evidence how amino acids and short peptides drive mutations, which is critical in predicting emergent strains and their deadly potential in the context of designing effective pan-vaccines and preventing the spread of disease. Some studies have found evidence that coevolving amino acids play a pivotal role in increasing the affinity of the spike protein against ACE2, leading to more successful infection, with some of these amino acids under more evolutionary pressure than others [3]. On the other hand, other viral proteins, such as the non-structural protein 1, contribute to immune evasion. The coevolution impact on interaction patterns of proteins among a growing number of variants should not be neglected. Short peptides are naturally suited to treating infectious disease as they can disrupt protein-protein interactions [4]. Notably, these inter-contacts are the heart of most important cellular processes and primary targets for smart drug discovery but are "undruggable" by small-molecules. Notably, there are a plethora of disease-relevant proteinprotein interactions, but most of them have so far been unexplored. More specifically, viral proteins take over cellular host functions through short peptide interaction motifs (in unstructured regions) that bind to defined pockets on globular host domains. These motifs evolve by mutation, enabling viruses to interact with novel host factors. An understanding of these peptide-mediated protein-protein interactions can predict viral tropism and molecular processes within host cells. They could be targets for novel antiviral inhibitors, such as integrin-targeted drugs in controlling COVID-19 [5]. The design of suitable antiviral drugs became possible by thorough knowing the composition of the binding site pocket of virus (SARS-CoV-2) main protease [6]. Structural mapping a protein network, that enables studying suitable protein interactions with other proteins, can reveal repurposed drugs that could target disease relevant processes. This new tactic has identified cyclic depsipeptide, a known anticancer drug, which is more potent than remdesivir in COVID-19. This idea could be used for other pathogens [7]. The concept of smart vaccines using machine learning, structural modelling, to precisely predict the binding between viral peptides and host proteins from the adaptive immune system or other evolutionary peptides, leading to an increase in the speed of vaccine development in future health crises [8]. Thus, advanced structural biology is a valuable tool to gather information helpful in controlling current and next outbreaks of deadly pathogens as well as rapid progress in the development of more effective drugs and vaccines. 
[1] Bárcena, M., Barnes, C.O., Beck, M. et al. (2021). Nat. Struct. Mol. Biol., $28,2$.

[2] Lynch, M.L., Snell, E.H., Bowman, S.E.J. (2021). IUCrJ 8, 335.

[3] Priya, P., Shanker, A. (2021). Infect, Genet. \& Evol. 87, 104646.

[4] Apostolopoulos, V., Bojarska, J., Chai, T.T. et al. (2021). Molecules 26, 430.

[5] Kruse, T., Benz, C., Garvanska, D.H. et al. (2021). bioRxiv 19,10.1101/2021.04.19.440086

[6] Dai, W., Zhang, B., Jiang, X.M. et al. (2020). Science, 368, 1331.

[7] White, K.M., Rosales, R., Yildiz, S. et al. (2021). Science, 371, 926.

[8] Alam, A,. Khan, A., Imam, N. et al. (2021). Briefings in Bioinformatics, 22, 1309.

\section{Keywords: short peptides; structural biology; pandemic}

